\title{
An algebraic method for eye blink artifacts detection in single channel EEG recordings
}

\author{
Z. Tiganj ${ }^{1}$, M. Mboup ${ }^{2}$, C. Pouzat $^{3}$ and L. Belkoura ${ }^{4}$ \\ ${ }^{1}$ Project ALIEN, INRIA Lille - Nord Europe, Villeneuve d'Ascq, France \\ 2 CReSTIC. Université de Reims Champagne Ardenne, Reims, France \\ ${ }^{3}$ Laboratoire de Physiologie Cérébral, Université Paris Descartes, Paris, France \\ ${ }^{4}$ LAGIS, Université des Sciences et Technologies de Lille, Lille, France
}

\begin{abstract}
Single channel EEG systems are very useful in EEG based applications where real time processing, low computational complexity and low cumbersomeness are critical constrains. These include brain-computer interface and biofeedback devices and also some clinical applications such as EEG recording on babies or Alzheimer's disease recognition. In this paper we address the problem of eye blink artifacts detection in such systems. We study an algebraic approach based on numerical differentiation, which is recently introduced from operational calculus. The occurrence of an artifact is modeled as an irregularity which appears explicitly in the time (generalized) derivative of the EEG signal as a delay. Manipulating such delay is easy with the operational calculus and it leads to a simple joint detection and localization algorithm. While the algorithm is devised based on continuous-time arguments, the final implementation step is fully realized in a discrete-time context, using very classical discrete-time FIR filters. The proposed approach is compared with three other approaches: (1) the very basic threshold approach, (2) the approach that combines the use of median filter, matched filter and nonlinear energy operator (NEO) and (3) the wavelet based approach. Comparison is done on: (a) the artificially created signal where the eye activity is synthesized from real EEG recordings and (b) the real single channel EEG recordings from 32 different brain locations. Results are presented with Receiver Operating Characteristics curves. The results show that the proposed approach compares to the other approaches better or as good as, while having lower computational complexity with simple real time implementation. Comparison of the results on artificially created and real signal leads to conclusions that with detection techniques based on derivative estimation we are able to detect not only eye blink artifacts, but also any spike shaped artifact, even if it is very low in amplitude.
\end{abstract}

Keywords - Electroencephalography, eye blink artifacts, differential algebra, operational calculus, parameter estimation.

\section{PROBLEM DESCRIPTION}

In electroencephalographic (EEG) recordings electrical activity of the levator muscle (which controls the upper eyelid and is responsible for eye blinks), projects on vectors pitched by the EEG electrodes causing artifacts. Eye blink artifacts affect more then $10 \%$ of an average human EEG (human in average blinks once in $5 s$, and the duration of an artifact is more then $0.5 s$ ), thus they are a very important problem in EEG signal processing.

The amplitude of these artifacts depends on the amplitude of the levator muscle activity and on the brain region where the EEG is recorded. Typically, the amplitude has the same order of magnitude as the recorded brain activity. Thus, during the eye blink EEG recording is useless for most of applications. On the other hand, the artifacts can not be detected only by setting a threshold.

Common approach for eye blinks detection is placing extra electrodes close to the eye. Signal from these electrodes is amplified with differential amplifier, then setting an appropriate threshold is enough for the detection. Moreover, if several EEG channels are recorded, blind source separation is often used, not only to detect artifacts, but also to reject them from the EEG data .

However, placing extra electrodes close to the eye or using EEG with several channels is not suitable in many applications. Upcoming consumer application based on EEG such as brain-computer interface [1] or biofeedback devices [2] are mostly intended for non-clinical, everyday home use. Simplicity of use and low price are essential for those devices. Some clinical application such as recording on babies [3] or Alzheimer's disease recognition [4] are often based on single channel EEG systems as well. In this paper we address the problem of eye blink artifacts detection in such single channel systems. We show how the new algebraic technique for numerical differentiation [5] can lead to the algorithm for joint detection and estimation of the locations of the eye blink artifacts in a single channel recording.

In the following section we model the artifact-free part of 
the signal as a piecewise polynomial and we interpret the occurrence of a spike shaped artifact as a discontinuity point in that model. Next, we describe an algorithm which allows us to detect and locate such an irregularity point. The main steps of the algorithm, presented in section II, are based on the very classical operational calculus [6] (which is widely used in electrical circuit). The solution admits an easy implementation, as a combination of finite impulse response (FIR) filters.

In section III we compare the proposed approach with three other approaches: (1) the very basic threshold approach, (2) an approach that combines the use of median filter, matched filter and nonlinear energy operator (NEO) [7] and (3) the wavelet based spike detection algorithm [8]. Since the projection of the levator muscle activity depends on the brain region where EEG is recorded we did the comparison on single channel recordings from 32 different brain locations. To be able to have a complete control over the eye blink locations and the signal to noise ratio we also did a comparison of the four approaches on artificially created signal where we used eye blink templates obtained from the real EEG recordings. The results are presented with receiver operating characteristic (ROC) for each of the 32 locations. Representing results using ROC curves is standard in detection problems. They indicate that the proposed approach compares favorably with the threshold and NEO based approach, while it performs similarly as the wavelet approach, but it has significantly lower computational complexity and can be implemented for online detection.

\section{DESCRIPTION OF OUR APPROACH}

The association differential algebra-operational calculus leads to simple and efficient algebraic approach for parameter estimation, initiated in [6]. This approach is applied here in order to obtain an eye blink artifacts detection method. This can be seen as a change-point detection [9] problem: Given $y(t)$, a noisy observation of a piecewise regular signal $x(t)$, we want to detect the abrupt changes and estimate their locations. The problem is widely studied in the literature where the statistical approach naturally takes the dominant role (see [9], [10] and their references). Here, we apply a purely algebraic approach (see [6], [11] and [12] for the details).

We represent our signal with a piecewise polynomial model. The rapid variation induced by an eye blink artifact in interpreted as an irregular point in the signal. Consider an interval $I_{\tau}^{T}$, with the origin $\tau$ and length $T$. For $t \in I_{0}^{T}$, let set $x_{\tau}(t)=x(t+\tau)$. Next, choose $T$ such that there is at most one discontinuity point $t_{\tau}$ in $I_{\tau}^{T}$. Now, if $t_{\tau}$ is such an artifact location (an irregular point) then we know that the $n^{\text {th }}$ order derivative of $x(t)$ has the form:

$$
\frac{d^{n} x_{\tau}}{d t^{n}}=\left[x_{\tau}^{(n)}\right](t)+\sum_{k=0}^{n-1}\left(\mu_{k}^{0} \delta(t)^{(k)}+\mu_{k} \delta\left(t-t_{\tau}\right)^{(k)}\right),
$$

where the exponent $(k)$ stands for the derivative of order $k, \mu_{k}$ (resp. $\mu_{k}^{0}$ ) is the jump of the $k^{t h}$ order derivative at the point $t_{\tau}$ (resp. 0) and $[f]$ represents the regular part of $f$.

In a sequel we assume a piecewise polynomial model of order not exceeding 1 . Then, by taking $n=2$ in equation (1) the regular part, $\left[x_{\tau}^{(2)}\right](t)$, vanishes and we obtain:

$$
\frac{d^{2} x_{\tau}}{d t^{2}}=\mu_{0}^{0} \delta(t)+\mu_{1}^{0} \dot{\delta}(t)+\mu_{0} \delta\left(t-t_{\tau}\right)+\mu_{1} \dot{\delta}\left(t-t_{\tau}\right)
$$

To handle more easier expressions like $\delta(t)$, we rewrite equation (2) in the operational domain:

$$
s^{2} \hat{x}_{\tau}(s)=\mu_{0}^{0}+s \mu_{1}^{0}+\left(\mu_{0}+s \mu_{1}\right) e^{-t_{\tau} s} .
$$

As the jump parameters $\mu_{0}^{0}, \mu_{1}^{0}, \mu_{0}$ and $\mu_{1}$ are unknown we use the algebraic framework of [6] to eliminate them and obtain:

$$
t_{\tau}^{2}\left(s^{2} \hat{x}_{\tau}\right)^{(2)}+2 t_{\tau}\left(s^{2} \hat{x}_{\tau}\right)^{(3)}+\left(s^{2} \hat{x}_{\tau}\right)^{(4)}=0
$$

We apply the algorithm described in [13] (see also [14] and [15]) to obtain back in the time domain a second order equation in $t_{\tau}$ where the coefficients are iterated integrals of the signal. In the discrete time, we set $\tau=k T_{s}$ and $T=M T_{S}$ where $T_{s}$ is sampling time. Using classical numerical integration with sliding windows $I_{k}^{M}=\left[k T_{s}(k+M) T_{s}\right]$ we end up with a second order equation

$$
a_{k} t_{k}^{2}+b_{k} t_{k}+c_{k}=0
$$

where each coefficient is the output of a FIR filter, with the noisy observation as the input. In this equation we use the notation $t_{k}$ for the discrete counterpart of $t_{\tau}$. The detection will be based on the following observation [13]: If there is no discontinuity point in $I_{k}^{M}$ then all three coefficients of equation (5) are equal to 0 . Else these coefficients are all different from 0 . Therefore, as a detection criteria we use the product of the three coefficients: $Q_{k}=\left|a_{k} b_{k} c_{k}\right|$. Due to the noise, an eye blink artefact will be detected when $Q_{k}$ is greater than a given threshold $\sigma$, representing the noise level.

\section{RESULTS AND CONCLUSION}

In order to asses the efficiency of the proposed algebraic approach we compare it with three other approaches: 
- The basic threshold approach, where it is assumed that the eye blink artifacts have the largest amplitudes in the recorded signal and only a threshold is applied to detect them.

- The approach that combines median filtering, matched filtering and NEO. In the literature this approach is known to emphasize spike shape waveforms as the eye blinks [7].

- The approach that is based on wavelet decomposition, where scales are calculated depending on the input parameters: minimum and maximum spike width [8].

The comparison has been done on 7.5 minutes of 32 channel EEG recording, at the sampling frequency of $1 \mathrm{kHz}$. The electrodes were placed according to the international 10-20 system. The recording was unipolar with reference calculated as the averaged sum of all 32 electrodes. The ground electrode was placed on the forehead. All electrode impedances were less then $5 k \Omega$.

The parameter $\mathrm{T}$ is set to $0.5 \mathrm{~s}$, what gives us satisfying resolution since the duration of eye blink artifacts is typically longer than $0.5 \mathrm{~s}$.

Since we address the problem of the eye blink detection and localization in single channel EEG, all four approaches were applied on each of the 32 channels separately. Since the recorded signals are from different brain regions, we are able to evaluate how different electrodes positions affect the results of the different approaches.

In order to have reference information on when the examinee actually blinks, apart from the EEG, two additional electrodes were used. One was placed above and the other below the examinee's right eye. In 7.5 minutes of recording we detected 65 eye blinks. The results for each of the four approaches are presented with 32 ROC curves, one for each channel (fig. 1). Every curve is made from 50 points that are calculated for different threshold levels. The detection is taken as correct if the eye blink occurred in 1s window around the point that is found to be above the threshold level. For each of the 50 points, the probability of correct detection is defined as the ratio between the number of correct detections for a given threshold and the total number of spikes. The probability of false alarm is defined as the ratio between the number of false alarms and the maximal possible number of false alarms for a given threshold. From the results shown on fig. 1 we conclude the following:

- Since electrodes FP1 and FP2 are located very close to the eyes all four approaches have almost perfect detection rate (all eye blinks are detected with almost no false alarms).

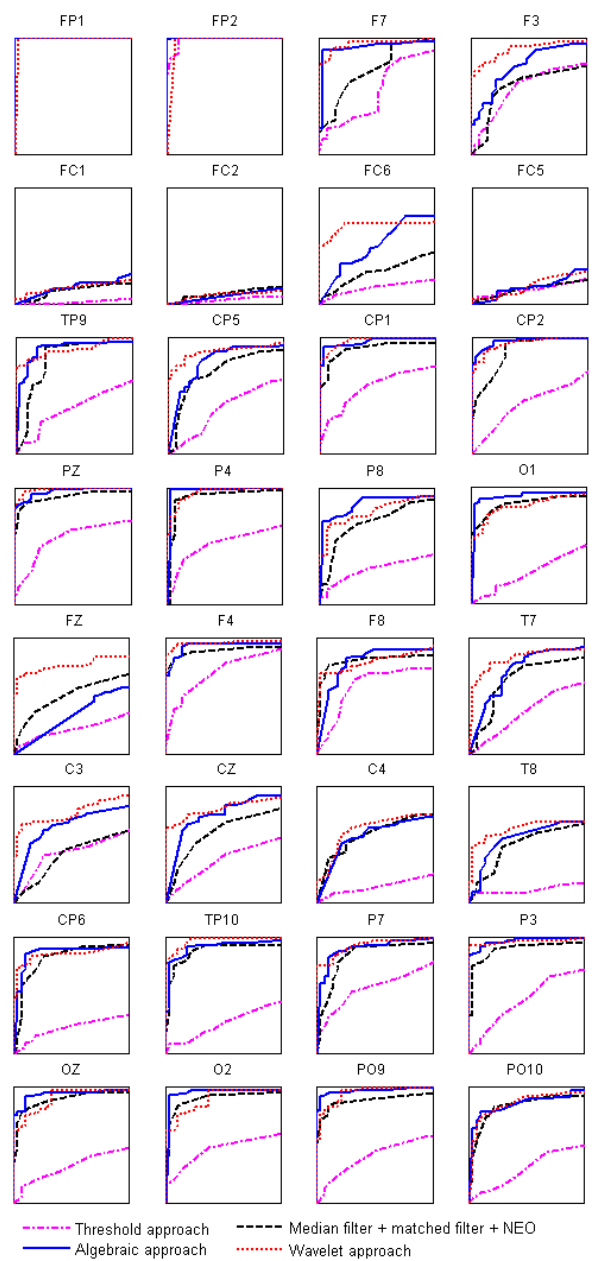

Fig. 1: 32 ROC curves obtained when real EEG signal is used. The vertical axis denotes probability of correct detection and goes from 0 to 1 , while the horizontal axis denotes probability of false alarm and goes from 0 to 0.03 .

- Poor detection results for channels FC5, FC1, FC2 and FC6 suggest that the projection of the eye muscle activity is very low in this region, what leads to conclusion that eye blink detection is not necessary in some cases.

- Advantages of using either algebraic approach, wavelet approach or combination of median filter, matched filter and NEO are the greatest when the algorithms are applied on signals from the electrodes that are placed further from the eyes (from TP9 to PO10 on fig. 1). Here we notice the property of the algorithms to emphasis spike shape waveforms in the noisy environment (since threshold approach gives poor results the recorded signal is obviously very noisy in these brain regions). For the electrodes F7, F3, $\mathrm{FZ}, \mathrm{F} 4$ and F8, where the projection of the eye activity is larger (since they are located closer to the eyes), threshold approach performs similar to the other three approaches. 
Simple to implement, the proposed algebraic approach allows extremely fast computation in comparison with the other approaches. The processing time (averaged from 50 iterations) of 7.5 minutes long input signal was 77.30 s for the NEO based approach, 3.22s for the algebraic approach (FIR filters with 500 samples long impulse responses were used) and 27.31s for the wavelet based approach (minimum spike width was set to $300 \mathrm{~ms}$ and maximum to $700 \mathrm{~ms}$, number of scales was set to 4 and biorthogonal mother wavelet was used). The implementation is done in MATLAB and run under Microsoft XP OS on the computer with $1.73 \mathrm{GHz}$ Intel Core2 processor and 2GB of RAM.

Simulation done on the real EEG recording gives a good overview of the algorithms performance on signals from different brain regions. However, this simulation has a drawback since we can not be sure that the eye artifacts are the only spike shaped artifacts in the signal. Any other muscle movement by the examinee or some electrodes shifting may cause such artifacts as well. Since we are not aware of these additional artifacts their detection by the algorithms will be considered as a false alarm.

In order to have a complete control over the artifacts locations we made an artificial signal by using white noise and randomly distributed eye blink template created by averaging eye blinks from channel $\mathrm{T} 8$ from the real EEG recording. The result of the comparison is again presented with ROC curve (fig.2). Now the algebraic and the wavelet approach performs similarly (while algebraic approach has much lower computational complexity) and better than approach based on combination of median filter, matched filter and NEO.

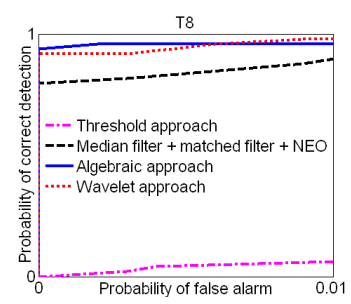

Fig. 2: ROC curve obtained when synthesized EEG signal is used.

The algebraic approach performs better with respect to the other approaches when simulation is done on the artificial signal (fig. 2) than when it is done on the real signal (fig. 1). This suggests that, as we mentioned earlier, the real signal had some other spike shape artifacts that we were not aware of and their detections were counted as false alarms.

Easy implementation (FIR filters), high robustness and the real time processing make the proposed approach quite eligible for eye artifact detection in single channel EEG systems. This is particularly interesting knowing that, due to low costs and simplicity of use, single channel devices become more and more common, especially in home use consumer devices, as well as in some clinical applications. Moreover this approach can also be applied in multichannel EEG and MEG systems.

\section{ACKNOWLEDGEMENTS}

The authors thank the University of Zagreb for providing them with the EEG.

\section{REFERENCES}

1. Thulasidas M, Guan C, Ranganatha S, Wu J K, Zhu X, Xu W. Effect of Ocular Artifact Removal in Brain Computer Interface Accuracy 26th Annual Inter Conference of the Engineering in Medicine and Biology Society. 2004;6:4385-4388.

2. Othmer S, Kaiser D. Implementation of Virtual Reality in EEG Biofeedback Cyberpsychology and Behavior. 2000;3:415-420.

3. Walls-Esquivel E, Vecchierini M F, Héberlé C, Wallois F. Electroencephalography (eeg) recording techniques and artefact detection in early premature babies Clinical Neurophysiology. 2007;37:299-309.

4. Cho S Y, Kim B Y, Park E H, et al. Automatic recognition of Alzheimer's disease with single channel EEG recording International Conference of the IEEE Engineering in Medicine and Biology. 2003;3:2655-2658.

5. Mboup M, Join C, Fliess M. Numerical differentiation with annihilators in noisy environment Numerical Algorithms. 2009;50:439-467.

6. Fliess M, Sira-Ramírez H. An algebraic framework for linear identification ESAIM: COCV. 2003;9:151-168.

7. Land B, Spence A. Spike (action potential) detection and sorting Journal Club 25, http://www.nbb.cornell.edu/neurobio/land/PROJECTS/spikeSort/. 2002.

8. Nenadic Z, Burdick J W. Spike detection using the continious wavelet transform IEEE Trans Biomed Eng. 2005;52:74-87.

9. Basseville M, Nikiforov I V. Detection of Abrupt Changes: Theory and Application Prentice-Hall. 1993.

10. Lavielle M. Using penalized contrasts for change-point problem Signal Processing. 2005;85:1501-1510.

11. Fliess M, Mboup M, Mounier H, Sira-Ramírez H. Questioning some paradigms of signal processing via concrete example. México: Editiorial Lagares 2003.

12. Mboup M. Parameter estimation for signals described by differential equations Applicable Analysis. 2009;88:29 - 52.

13. Mboup M. A Volterra filter for neuronal spike detection Research report, INRIA, http://hal inria fr/inria-00347048/fr/. 2008.

14. Fliess M, Join C, Mboup M. Algebraic change-point detection in press in AAECC, DOI:10.1007/s00200-010-0119z. 2010.

15. Tiganj Z, Mboup M. Spike Detection and Sorting: Combining Algebraic Differentiations with ICA in Independent Component Analysis and Signal Separation, 8th International Conference(Paraty, Brazil):475-482 2009

Author: Zoran Tiganj

Institute: INRIA Lille - Nord Europe

Street: Parc Scientifique de la Haute Borne 40

City: Villeneuve d'Ascq

Country: France

Email: zoran.tiganj@inria.fr 